\title{
Early Postemergence Herbicide Tank-Mixtures for Control of Waterhemp Resistant to Four Herbicide Modes of Action in Corn
}

\author{
Christian Willemse ${ }^{1}$, Nader Soltani ${ }^{*}$, Lauren Benoit ${ }^{1}$, Amit J. Jhala ${ }^{2}$, David C. Hooker ${ }^{1}$, \\ Darren E. Robinson', Peter H. Sikkema'
}

${ }^{1}$ Department of Plant Agriculture, University of Guelph, Ridgetown, Canada

${ }^{2}$ Department of Agronomy and Horticulture, University of Nebraska-Lincoln, Lincoln, NE, USA

Email: *soltanin@uoguelph.ca

How to cite this paper: Willemse, C., Soltani, N., Benoit, L., Jhala, A.J., Hooker, D.C., Robinson, D.E. and Sikkema, P.H. (2021) Early Postemergence Herbicide Tank-Mixtures for Control of Waterhemp Resistant to Four Herbicide Modes of Action in Corn. Agricultural Sciences, 12, 354-369.

https://doi.org/10.4236/as.2021.124023

Received: February 13, 2021

Accepted: April 6, 2021

Published: April 9, 2021

Copyright $\odot 2021$ by author(s) and Scientific Research Publishing Inc. This work is licensed under the Creative Commons Attribution International License (CC BY 4.0).

http://creativecommons.org/licenses/by/4.0/ (c) (i) Open Access

\begin{abstract}
Multiple-herbicide-resistant (MHR) waterhemp has been confirmed and is difficult to control for growers in Ontario, Canada and in the Midwestern United States. The objective of this study was to evaluate early post-emergence (EPOST) herbicides for control of MHR waterhemp in field corn. Five field trials were conducted over a two-year period $(2019,2020)$ at sites on Walpole Island, ON and near Cottam, ON, Canada. Thirteen herbicide tank-mixtures containing multiple modes-of-action (MOA) were applied EPOST to $5 \mathrm{~cm}$ MHR waterhemp in field corn. Control of MHR waterhemp varied by site due to variable plant density, plant biomass, and number of herbicide-resistant individuals across research sites and years. Control of MHR waterhemp ranged from $90 \%$ to $100 \%$ with glyphosate $+S$-metolachlor/mesotrione/ bicyclopyrone/atrazine, glyphosate/2,4-D choline + rimsulfuron + mesotrione + atrazine, glyphosate $+S$-metolachlor/atrazine/mesotrione, glyphosate + mesotrione + atrazine, glyphosate $/ S$-metolachlor/mesotrione + atrazine, glyphosate $+S$-metolachlor/mesotrione/bicyclopyrone, glyphosate/2,4-D choline + rimsulfuron + mesotrione, and glyphosate + pyroxasulfone + dicamba/atrazine at 4, 8, and 12 WAA. Control of MHR waterhemp ranged from $70 \%$ to $100 \%$ with glyphosate + topramezone/dimethenamid-P + dicamba/atrazine, glyphosate + isoxaflutole + atrazine, and glyphosate + tolpyralate + atrazine at 4, 8, and 12 WAA. Control of MHR waterhemp was similar for all herbicide programs, except glyphosate + dicamba/atrazine and glyphosate $+S$-metolachlor/atrazine which resulted in the lowest control at three of five sites that ranged from $63 \%$ to $89 \%$ and $61 \%$ to $76 \%$, respectively. Crop injury was $\leq 10 \%$ for herbicide programs tested, except $28 \%$ to $31 \%$ corn injury with glyphosate/2,4-D choline + rimsulfuron + mesotrione + atrazine; however,
\end{abstract}


without effect on corn grain yield. Corn yield was comparable with all herbicide programs evaluated in this study. It is concluded that there are herbicide programs that provide control of emerged and full-season residual control of MHR waterhemp in field corn.

\section{Keywords}

Density, Biomass, Residual Weed Control, Weed Management

\section{Introduction}

Waterhemp [Amaranthus tuberculatus (Moq.) J. D. Sauer] has become one of the most problematic weed species in midwestern United States crop production. Reductions in tillage, greater reliance on herbicides for weed management, and the evolution of resistance to multiple herbicide modes of action (MOA) have contributed to the rapid increase of waterhemp in agricultural cropping systems [1] [2]. Waterhemp has been reported in 19 states of the USA and three provinces in Canada where it interferes with corn and soybean production [3] [4]. The rapid movement of waterhemp and evolution of herbicide resistance among individuals and populations is facilitated by its dioecism, rapid growth rate, high reproductive rate, delayed emergence, and extended emergence pattern [5] [6]. Resistance to photosystem II (PS II)-, acetolactate synthase (ALS)-, and protoporphyrinogen oxidase (PPO)-inhibiting herbicides was identified in waterhemp in 1990, 1993, and 2001, respectively [4] [7] [8] [9]. Waterhemp resistant to 5-enolpyruyl shikimate-3-phosphate synthase (EPSPS)-inhibitors was first reported in the USA in 2005 and Ontario, Canada in 2014 [4]. More recent reports from Ontario have identified multiple-herbicide-resistant (MHR) waterhemp populations resistant to ALS-, PS II-, EPSPS-, and PPO-inhibiting herbicides. Waterhemp continues to evolve resistance to currently used MOA and is the first weed species to develop resistance to 4-hydroxyphenylpyruvate dioxygenase (HPPD)-inhibiting herbicides [4] [7] [8] [9]. The first MHR waterhemp population with six-way resistance to synthetic auxins and ALS-, PS II-, EPSPS-, PPO-, and HPPD-inhibiting herbicides was identified in Missouri in 2015 [10]. Resistance to very-long-chain fatty-acid (VLCFA)-inhibiting herbicides has since been detected within a MHR waterhemp population resistant to 2,4-D and ALS-, PS II-, PPO-, and HPPD-inhibiting herbicides [11]. The ability of MHR waterhemp to rapidly evolve and accumulate traits that confer resistance to multiple MOA makes it difficult to manage in agricultural cropping systems [12].

Weed interference must be prevented during the early stages of corn (Zea mays L.) growth and development to prevent yield loss [13]. The relative time of crop and weed emergence has a greater effect on corn yield than weed density and biomass [13]. Weeds that emerge with the crop have the greatest impact on corn yield [13]. Steckel and Sprague [2] reported MHR waterhemp emerging at 
VE corn growth stage reduced grain yield $74 \%$ compared to only $2 \%$ yield loss when waterhemp emerged at V8 and was left uncontrolled for the remainder of the growing season. In contrast, Cordes et al. [14] reported corn yield loss was dependent on waterhemp density. When competing with corn, waterhemp can be placed at a disadvantage due to its characteristic late emergence; however, corn yield losses of up to $17 \%$ have been reported when densities of 369 to 445 plants $\mathrm{m}^{-2}$ emerge and compete up to V7 corn growth stage [14] [15]. Steckel and Sprague [2] reported corn yield reductions when waterhemp emerged before the V8 corn growth stage. In Ontario, corn yield losses of up to $48 \%$ have been reported when waterhemp populations are left uncontrolled [16]. The critical period of weed control in corn to prevent yield loss varies with the relative time of weed and crop emergence, weed density, species, and environment [17] [18]. It is recommended that corn remain waterhemp-free from emergence to V6 to maximize grain yield [2]. Early-season control of MHR waterhemp is imperative to reduce early-season weed interference, prevent corn yield loss, and reduce weed escapes.

Current herbicide-based MHR waterhemp management strategies include preemergence (PRE), postemergence (POST), and PRE followed-by ( $f b$ ) POST herbicide applications that utilize multiple effective MOA [16] [19] [20] [21]. The HPPD-inhibitors isoxaflutole, mesotrione, and tolpyralate are often applied in combination with a PS II-inhibitor such as atrazine and result in excellent control of MHR waterhemp [22] [23] [24] [25] [26]. Complementary activity between HPPD-inhibitors and atrazine has been reported for the control of triazine-susceptible and triazine-resistant redroot pigweed (Amaranthus retroflexus L.), waterhemp, and Palmer amaranth (Amaranthus palmeri S. Watson) [25] [26] [27]. HPPD-inhibitors inhibit the production of carotenoids, $\alpha$-tocopherols, and plastoquinone, and atrazine increases the production of reactive oxygen species [28] [29]. The enhanced weed control efficacy when a HPPD-inhibitor is co-applied with a PS II-inhibitor is due to 1) increased binding efficiency of atrazine to the D1 protein of PS II-inhibitor caused by the shortage of plastoquinone, and 2) enhanced reactive oxygen species (ROS) levels due to the lack of quenching carotenoids, tocopherols, and plastoquinone. Synthetic auxin herbicides are another effective MOA for MHR waterhemp control; however, current literature reports variable responses [16] [30] [31]. Synthetic auxin herbicides provide control of broadleaf weeds by mimicking plant growth hormones which causes unregulated plant growth and death in some plants [32]. Superior MHR waterhemp control with dicamba/atrazine compared to other POST tank-mixtures has been reported [33]. Anderson et al. [30], Soltani et al. [16] and Vyn et al. [31] reported dicamba/atrazine provided $\geq 86 \%$ control of herbicide-resistant waterhemp. Benoit et al. [33] and Schryver et al. [34] found that POST applications of dicamba are more effective than PRE applications. The application of a new glyphosate/2,4-D choline formulation registered for application to ENLIST $^{\mathrm{TM}}$ (Corteva Agriscience, Wilmington, DE) corn hybrids allows for a 
second synthetic auxin herbicide for MHR waterhemp control in corn [34] [35] [36]. ENLIST ${ }^{\mathrm{TM}}$ corn hybrids contain transgenes that confer resistance to glyphosate and glufosinate plus the aryloxyalkanoate dioxygenase-1 (AAD-1) transgene which enables them to exhibit resistance to glyphosate, glufosinate, and greater tolerance to 2,4-D and the arlyloxyphenoxy propionates than traditional glyphosate (RoundupReady ${ }^{\circledR}$ ) (Bayer CropScience Inc., 160 Quarry Park Boulevard SE, Calgary, AB) and glufosinate (LibertyLink ${ }^{\circledR}$ ) (BASF Canada Inc., 100 Milverton Drive, Mississauga, ON) resistant hybrids [35] [37] [38]. Robinson et al. [36] reported up to $94 \%$ control of common waterhemp (Amaranthus rudis Sauer) with 2,4-D (1120 g ae) and 99\% control with 2,4-D + glyphosate $(280+840 \mathrm{~g}$ ae) ; however, it is important to note glyphosate (840 $\mathrm{g}$ ae) alone provided $100 \%$ control 4 WAA in that study. Similarly, Miller and Norsworthy [39] obtained $\geq 87 \%$ control of glyphosate-resistant Palmer amaranth in soybean. Numerous PRE, POST, and PRE $f b$ POST herbicide applications in corn have been developed for the control of MHR waterhemp with varying resistance profiles [3] [19] [31] [33] [40] [41].

Waterhemp control is affected by herbicide application timing. Previous studies have shown that PRE applications can control MHR waterhemp better than POST applications [33] [42] [43]. Hager et al. [44] reported $8 \%$ to $9 \%$ greater control of ALS-resistant-waterhemp 1, 2, and 3 WAA when herbicides were applied to $5 \mathrm{~cm}$ waterhemp early POST (EPOST) compared to $10 \mathrm{~cm}$ waterhemp (POST) in soybean. Similarly, Hedges et al. [43] observed a $20 \%$ reduction in waterhemp control as POST applications were delayed from 5 to $25 \mathrm{~cm}$ tall waterhemp. These studies suggest differences between EPOST and POST can be attributed to slower herbicide activity on larger waterhemp plants and reduced interception due to shading of younger plants caused by the extended emergence pattern [33] [42] [43]. Corn producers should eliminate MHR waterhemp interference from VE to V6 corn growth stage and control it before it exceeds $10 \mathrm{~cm}$ in height [2] [44] [45].

Delayed POST herbicide applications can result in reduced control due to larger weed size at application and decreased corn yield due to early-season waterhemp interference. To achieve season-long control of MHR waterhemp, it is imperative that herbicide applications include effective MOA, provide soil residual and target small weed size $(\leq 10 \mathrm{~cm})$. We hypothesized that EPOST herbicide tank mixtures made to $5 \mathrm{~cm}$ MHR waterhemp will provide season-long control of MHR waterhemp in corn. The objective of this research was to identify effective EPOST herbicide tank-mixtures that provide control of emerged MHR waterhemp and season-long residual control in corn while stewarding currently available herbicide MOA.

\section{Materials and Methods}

\subsection{Experimental Methods}

Five field trials were conducted over a two-year period $(2019,2020)$ at sites on 
Walpole Island, $\mathrm{ON}\left(42.561492^{\circ} \mathrm{N},-82.501487^{\circ} \mathrm{W}\right)$ and near Cottam, ON, Canada $\left(42.149076^{\circ} \mathrm{N},-82.683687^{\circ} \mathrm{W}\right)$ with $\mathrm{MHR}$ waterhemp resistant to ALS-, PS II-, EPSPS- and PPO-inhibiting herbicides (Table 1). Sites were disked or cultivated in the spring to prepare the seedbed for planting. Glyphosate- and glufosinate-resistant corn hybrid DKC45-65RIB (Monsanto, St. Louis, MO) was seeded in rows spaced $0.75 \mathrm{~m}$ apart at approximately 83,000 seeds ha ${ }^{-1}$ to a depth of 4 $\mathrm{cm}$. Plots were $8 \mathrm{~m}$ long and $2.25 \mathrm{~m}$ ( 3 corn rows) wide. Fifteen herbicide treatments (Table 2) were arranged in a randomized complete block design with four replications. Replications included nontreated and weed-free controls and were separated by a $2 \mathrm{~m}$ alley. The weed-free control was maintained weed-free with a pre-emergence (PRE) application of atrazine/bicyclopyrone/mesotrione/ $S$-metolachlor (2022 $\left.\mathrm{g} \cdot \mathrm{ha}^{-1}\right)$ followed by either atrazine/dicamba (1800 $\left.\mathrm{g} \cdot \mathrm{ha}^{-1}\right)$ applied postemergence (POST) up to V3-stage (5-leaf stage) of corn development, or glufosinate (500 $\mathrm{g} \cdot \mathrm{ha}^{-1}$ ) between V3 and V6; hand-weeding was performed throughout the remainder of the growing season as needed. Glyphosate (450 $\mathrm{g}$ ae $\mathrm{ha}^{-1}$ ) was applied POST to the entire experimental area, including the nontreated control, to remove susceptible waterhemp biotypes and other weed species.

Herbicide treatments were applied EPOST using a $\mathrm{CO}_{2}$-powered backpack sprayer equipped with four, 120-02 ultra low drift (ULD) nozzles (Pentair, New Brighton, MN) spaced $50 \mathrm{~cm}$ apart and calibrated to deliver $200 \mathrm{~L} \cdot \mathrm{ha}^{-1}$ at 240 $\mathrm{kPa}$. All herbicide treatments were applied when MHR waterhemp reached an average $5 \mathrm{~cm}$ in height. Site 1 (S1) and S3 was separated temporally by applying herbicide treatments 5 days apart.

Data were collected on MHR waterhemp control estimates, density, biomass, visible corn injury, grain corn moisture content, and grain corn yield. Waterhemp control was evaluated visually on a $0 \%$ to $100 \%$ scale compared to the nontreated control at 4, 8, and 12 WAA. MHR waterhemp density and biomass were determined at $4 \mathrm{WAA}$ by counting and harvesting the plants within two randomly placed $0.25 \mathrm{~m}^{2}$ quadrats in each plot. The aboveground biomass of the plants within each quadrat was determined by cutting the MHR waterhemp at

Table 1. Soil characteristics and resistance profile of each field site where herbicide tank-mixtures were applied EPOST for control of multiple-herbicide resistant (MHR) waterhemp in Ontario, Canada in 2019 and 2020.

\begin{tabular}{ccccccccccccc}
\hline & \multicolumn{1}{c}{} & \multicolumn{4}{c}{ Soil characteristics } & \multicolumn{4}{c}{ Resistance profile $^{\mathrm{a}}$} \\
\hline Site & Year & Location & Classification & Sand (\%) & Silt (\%) & Clay (\%) & pH & OM (\%) & ALS (\%) & PS II (\%) EPSPS (\%) & PPO (\%) \\
\hline S1 & 2019 & Cottam & Sandy Loam & 70 & 21 & 9 & 6.0 & 2.6 & 97 & 34 & N/A & N/A \\
S2 & 2019 & Walpole & Sandy Loam & 70 & 21 & 9 & 7.6 & 2.3 & 23 & 6 & 79 & N/A \\
S3 & 2019 & Cottam & Sandy Loam & 70 & 21 & 9 & 6.0 & 2.6 & 97 & 34 & N/A & N/A \\
S4 & 2020 & Cottam & Sandy Loam & 70 & 19 & 11 & 5.9 & 2.6 & 68 & 54 & 64 & 43 \\
S5 & 2020 & Walpole & Sandy Loam & 76 & 15 & 9 & 7.8 & 2.5 & 54 & 30 & 96 & 17 \\
\hline
\end{tabular}

Abbreviations: ALS, acetolactate synthase; OM, organic matter; PS II, photosystem II; EPSPS, 5-enolpyruvylshikimate-3-phosphate synthase; PPO, protoporphyrinogen oxidase. ${ }^{a}$ Mean number of surviving waterhemp plants three weeks after application divided by the number of plants sprayed within quadrats per mode of action per site. 
Table 2. Herbicide treatments, products, rates, and manufacturers for the study of herbicide tank-mixtures applied EPOST for the control of multiple-herbicide resistant (MHR) waterhemp in Ontario, Canada in 2019 and 2020.

\begin{tabular}{|c|c|c|c|}
\hline Herbicide treatment & Herbicide trade name & Rate (g ae or ai ha ${ }^{-1}$ ) & Herbicide manufacturer $^{\mathrm{b}}$ \\
\hline Glyphosate $+S$-metolachlor/atrazine & $\begin{array}{l}\text { Roundup WeatherMAX }{ }^{\circledR}+\text { Primextra }^{\circledR} \text { II } \\
\text { Magnum }^{\circledR}\end{array}$ & $900+1600 / 1280$ & $\begin{array}{l}\text { Bayer CropScience/Syngenta } \\
\text { Canada }\end{array}$ \\
\hline Glyphosate + dicamba/atrazine & $\begin{array}{l}\text { Roundup WeatherMAX }{ }^{\circledR}+\text { Marksman }^{\circledR} \\
\text { Herbicide }\end{array}$ & $900+504 / 996$ & $\begin{array}{l}\text { Bayer CropScience/BASF } \\
\text { Canada. }\end{array}$ \\
\hline Glyphosate + tolpyralate + atrazine & $\begin{array}{l}\text { Roundup WeatherMAX }{ }^{\circledR}+\text { Shieldex }^{\mathrm{TM}} 400 \\
\text { SC Herbicide + Aatrex }{ }^{\circledR}\end{array}$ & $900+40+1120$ & $\begin{array}{l}\text { Bayer CropScience/ISK } \\
\text { Biosciences }\end{array}$ \\
\hline Glyphosate + isoxaflutole + atrazine & $\begin{array}{l}\text { Roundup WeatherMAX }{ }^{\circledR}+\text { Converge Flexx } \\
\text { Herbicide + Aatrex }{ }^{\circledR} \text { Liquid } 480\end{array}$ & $900+105+1063$ & $\begin{array}{l}\text { Bayer CropScience/Bayer } \\
\text { CropScience/Syngenta Canada }\end{array}$ \\
\hline $\begin{array}{l}\text { Glyphosate + topramezone/ } \\
\text { dimethenamid-P + dicamba/atrazine }\end{array}$ & $\begin{array}{l}\text { Roundup WeatherMAX }{ }^{\circledR}+\text { Armezon }^{\circledR} \text { PRO } \\
+ \text { Marksman }{ }^{\circledR} \text { Herbicide }\end{array}$ & $900+12.5 / 630+488 / 966$ & $\begin{array}{l}\text { Bayer CropScience/BASF } \\
\text { Canada/BASF Canada }\end{array}$ \\
\hline $\begin{array}{l}\text { Glyphosate + pyroxasulfone + } \\
\text { dicamba/atrazine }\end{array}$ & $\begin{array}{l}\text { Roundup WeatherMAX }{ }^{\circledR}+\text { Zidua }^{\mathrm{TM}} \mathrm{SC}+ \\
\text { Marksman }{ }^{\circledR} \text { Herbicide }\end{array}$ & $900+150+488 / 966$ & $\begin{array}{l}\text { Bayer CropScience/BASF } \\
\text { Canada/BASF Canada }\end{array}$ \\
\hline $\begin{array}{l}\text { Glyphosate/2,4-D choline + rimsulfuron } \\
+ \text { mesotrione }\end{array}$ & $\begin{array}{l}\text { Roundup WeatherMAX }{ }^{\circledR}+\text { Enlist Duo } \\
\text { Herbicide }+ \text { Matrix }^{\circledR} \text { SG }+ \text { Callisto }^{\circledR} 480 S C \\
\text { Herbicide }\end{array}$ & $563 / 591+15+144$ & $\begin{array}{l}\text { Bayer CropScience/Corteva } \\
\text { Agriscience/Corteva } \\
\text { Agriscience/Syngenta Canada }\end{array}$ \\
\hline $\begin{array}{l}\text { Glyphosate }+S \text {-metolachlor/mesotrione/ } \\
\text { bicyclopyrone }\end{array}$ & Roundup WeatherMAX ${ }^{\circledR}+$ Acuron $^{\circledR}$ Flexi & $900+1268 / 141 / 35$ & $\begin{array}{l}\text { Bayer CropScience/Syngenta } \\
\text { Canada }\end{array}$ \\
\hline $\begin{array}{l}\text { Glyphosate/ } S \text {-metolachlor/mesotrione + } \\
\text { atrazine }\end{array}$ & $\begin{array}{l}\text { Roundup WeatherMAX }{ }^{\circledR}+\text { Halex }^{\circledR} \text { GT } \\
\text { Herbicide }\end{array}$ & $1050 / 1050 / 105+280$ & $\begin{array}{l}\text { Bayer CropScience/Syngenta } \\
\text { Canada }\end{array}$ \\
\hline Glyphosate + mesotrione + atrazine & $\begin{array}{l}\text { Roundup WeatherMAX }{ }^{\circledR}+\text { Callisto }^{\circledR} 480 S C \\
\text { Herbicide + Aatrex }{ }^{\circledR} \text { Liquid } 480\end{array}$ & $900+100+280$ & $\begin{array}{l}\text { Bayer CropScience/Syngenta } \\
\text { Canada/Syngenta Canada }\end{array}$ \\
\hline $\begin{array}{l}\text { Glyphosate }+ \\
S \text {-metolachlor/atrazine/mesotrione }\end{array}$ & $\begin{array}{l}\text { Roundup WeatherMAX }{ }^{\circledR}+\operatorname{Lumax}^{\circledR} \text { EZ } \\
\text { Herbicide }\end{array}$ & $900+1393 / 524 / 139$ & $\begin{array}{l}\text { Bayer CropScience/Syngenta } \\
\text { Canada }\end{array}$ \\
\hline $\begin{array}{l}\text { Glyphosate/2,4-D choline + rimsulfuron } \\
\text { + mesotrione + atrazine }\end{array}$ & $\begin{array}{l}\text { Roundup WeatherMAX }{ }^{\circledR}+\text { Enlist } \text { Duo }^{\mathrm{TM}} \\
{\text { Herbicide }+ \text { Matrix }^{\circledR} \mathrm{SG}+\text { Callisto }}^{\circledR} 480 \mathrm{SC} \\
\text { Herbicide + Aatre }{ }^{\circledR} \text { Liquid } 480\end{array}$ & $563 / 591+15+144+100$ & $\begin{array}{l}\text { Bayer CropScience/Corteva } \\
\text { Agriscience/Corteva } \\
\text { Agriscience/Syngenta } \\
\text { Canada/Syngenta Canada }\end{array}$ \\
\hline $\begin{array}{l}\text { Glyphosate }+S \text {-metolachlor/mesotrione/ } \\
\text { bicyclopyrone/atrazine }\end{array}$ & $\begin{array}{l}\text { Roundup WeatherMAX }{ }^{\circledR}+\text { Acuron }^{\circledR} \\
\text { Herbicide }\end{array}$ & $900+588 / 35 / 140 / 1259$ & $\begin{array}{l}\text { Bayer CropScience/Syngenta } \\
\text { Canada }\end{array}$ \\
\hline
\end{tabular}

Note: Herbicide treatments glyphosate + mesotrione + atrazine and glyphosate $/ S$-metolachlor/mesotrione + atrazine included Agral ${ }^{\circledR} 90$ (Syngenta Canada Inc., 140 Research Lane, Research Park, Guelph, ON.) (0.2\% v/v); and glyphosate + tolpyralate + atrazine included methylated seed oil (MSO Concentrate ${ }^{\circledR}$ ) (Loveland Products Inc., 3005 Rocky Mountain Ave., Loveland, CO) (0.5\% v/v), and urea ammonium nitrate (UAN 28-0-0) (Sylvite, 3221 North Service Road, Burlington, ON) (2.5\% v/v). ${ }^{a} \mathrm{DMA}$, dimethylamine. ${ }^{\mathrm{b} B A S F}$ Canada Inc., 100 Milverton Drive, Mississauga, ON; Bayer CropScience Inc., 160 Quarry Park Boulevard SE, Calgary, AB; Corteva Agriscience, 735 Chestnut Run Plaza, Wilmington, DE; ISK Biosciences Corporation, 7470 Auburn Road, Concord, OH; Syngenta Canada Inc., 140 Research Lane, Research Park, Guelph, ON).

the soil surface, the plants placed inside a paper bag, kiln-dried for three weeks to a consistent moisture, then weighed using an analytical balance to calculate MHR waterhemp biomass per unit area $\left(\mathrm{g} \cdot \mathrm{m}^{-2}\right)$. Visible corn injury was assessed on a $0 \%$ to $100 \%$ scale at 1 and 4 weeks after herbicide application (WAA); $0 \%$ represented no visible injury and $100 \%$ represented complete plant death. Grain corn yield $\left(\mathrm{t} \cdot \mathrm{ha}^{-1}\right)$ and moisture (\%) were collected by harvesting two rows of each plot at maturity using a small-plot combine. Grain yields were adjusted to $15.5 \%$ moisture prior to statistical analysis. 


\subsection{Statistical Analysis}

Data were subjected to variance analysis using the PROC GLIMMIX procedure in SAS v. 9.4 (SAS Institute Inc., Car, NC). An initial mixed model analysis was conducted to evaluate site-by-treatment interactions. Site, site-by-treatment, and replication within site were considered the random effect and the fixed effect was treatment. Site-by-treatment interaction was significant for all parameters with no difference between S1 and S3, and S2 and S5; therefore, data were combined for S1 and S3, and S2 and S5, and are presented separately for S4. A second mixed model analysis was conducted to analyze herbicide treatment effects on visible MHR waterhemp control, density, biomass, visible corn injury, and grain corn yield. The fixed effect was herbicide treatment and the random effect was replication. Normality and homogeneity of variance were tested using the Shapiro-Wilk test via the PROC UNIVARIATE procedure. Normality assumptions (residuals are independent, homogeneous and normally distributed) were confirmed by plotting the residuals for treatment, replication, and site. A normal distribution was used to analyze visible MHR waterhemp control, visible crop injury, and corn yield data. MHR waterhemp density and biomass data were analyzed using a lognormal distribution to satisfy assumptions of variance analysis. MHR waterhemp density and biomass least-square means were back-transformed from the log-scale using the omega method (M. Edwards, Ontario Agricultural College Statistician, University of Guelph, personal communication). Means were separated using the Tukey-Kramer grouping for Least Square Means. A significance level of $\alpha=0.05$ was used for data analysis.

\section{Results and Discussion}

Most EPOST herbicide tank-mixtures provided greater than $90 \%$ control of MHR waterhemp. The density, biomass, and population resistance profile are reflected in the differences in control between sites. At 4, 8, and 12 WAA, control of MHR waterhemp ranged from $61 \%$ to $100 \%$ across sites and was lower at S1, S3, and S4 due to greater density and biomass compared to S2 and S5 (Table 1 , Tables 3-5). Density and biomass of MHR waterhemp in the nontreated control at S1, S3, and S4 averaged 263 to 962 plants $\mathrm{m}^{-2}$ and 70.2 to $259.4 \mathrm{~g} \cdot \mathrm{m}^{-2}$, respectively, compared to 60 plants $\mathrm{m}^{-2}$ and $72.2 \mathrm{~g} \cdot \mathrm{m}^{-2}$ at $\mathrm{S} 2$ and S5. Vyn et al. [31] reported similar site differences in POST MHR waterhemp control which they attributed to plant density and site-specific MHR waterhemp resistance profiles. In that study, one waterhemp population exhibited resistance to ALS-inhibiting herbicides and the other to both ALS- and PS II-inhibiting herbicides [31]. All sites contained waterhemp resistant to ALS-, PS II-, EPSPS- and PPO-inhibitors; however, the proportion of individuals resistant to each MOA varied by site. The MHR waterhemp population at S1, S3, and S4 contained a greater number of individuals exhibiting resistance to ALS-, PS II-, and PPO-inhibitors than that of S2 and S5. 
Table 3. Means for multiple-herbicide-resistant (MHR) waterhemp control [4, 8 and 12 weeks after EPOST application (WAA)] in corn treated with herbicide tank-mixtures applied EPOST from five field trials conducted in Ontario, Canada in 2019 and 2020.

\begin{tabular}{|c|c|c|c|c|c|c|c|c|c|c|}
\hline \multirow[b]{2}{*}{ Treatment $^{\dagger}$} & \multirow[b]{2}{*}{ Rate (g ae or ai ha ${ }^{-1}$ ) } & \multicolumn{9}{|c|}{ Visible control (\%) } \\
\hline & & \multicolumn{3}{|c|}{4 WAA } & \multicolumn{3}{|c|}{8 WAA } & \multicolumn{3}{|c|}{12 WAA } \\
\hline Weed-free control & - & 100 & 100 & 100 & 100 & 100 & 100 & 100 & 100 & 100 \\
\hline Nontreated control & - & 0 & 0 & 0 & 0 & 0 & 0 & 0 & 0 & 0 \\
\hline Glyphosate $+S$-metolachlor/atrazine & $900+1600 / 1280$ & $75 \mathrm{~b}$ & $73 \mathrm{c}$ & $100 \mathrm{ab}$ & $67 \mathrm{bc}$ & $73 \mathrm{c}$ & $100 \mathrm{a}$ & $61 \mathrm{c}$ & $76 \mathrm{c}$ & $100 \mathrm{a}$ \\
\hline Glyphosate + dicamba/atrazine & $900+504 / 996$ & $76 \mathrm{~b}$ & $83 \mathrm{bc}$ & $99 \mathrm{~b}$ & $65 c$ & $86 \mathrm{~b}$ & $99 \mathrm{~b}$ & $63 c$ & $89 \mathrm{~b}$ & $99 \mathrm{~b}$ \\
\hline Glyphosate + tolpyralate + atrazine & $900+40+1120$ & $95 \mathrm{a}$ & 99 a & $100 \mathrm{ab}$ & $83 \mathrm{ab}$ & 99 a & $100 \mathrm{a}$ & $79 \mathrm{bc}$ & 99 a & $100 \mathrm{a}$ \\
\hline Glyphosate + isoxaflutole + atrazine & $900+105+1063$ & $92 \mathrm{a}$ & $94 \mathrm{ab}$ & $100 \mathrm{a}$ & $87 \mathrm{a}$ & $95 \mathrm{ab}$ & $100 \mathrm{a}$ & $84 \mathrm{ab}$ & $96 \mathrm{ab}$ & $100 \mathrm{a}$ \\
\hline Glyphosate + pyroxasulfone + dicamba/atrazine & $900+150+488 / 966$ & $95 \mathrm{a}$ & $93 \mathrm{ab}$ & $100 \mathrm{ab}$ & $92 \mathrm{a}$ & $95 \mathrm{ab}$ & $100 \mathrm{a}$ & $90 \mathrm{ab}$ & $97 \mathrm{ab}$ & $100 \mathrm{a}$ \\
\hline $\begin{array}{l}\text { Glyphosate/2,4-D choline + rimsulfuron + } \\
\text { mesotrione }\end{array}$ & $563 / 591+15+144$ & $98 \mathrm{a}$ & 99 a & $100 \mathrm{a}$ & $92 \mathrm{a}$ & 99 a & $100 \mathrm{a}$ & $90 \mathrm{ab}$ & 99 a & $100 \mathrm{a}$ \\
\hline $\begin{array}{l}\text { Glyphosate }+S \text {-metolachlor/mesotrione/ } \\
\text { bicyclopyrone }\end{array}$ & $900+1268 / 141 / 35$ & $95 \mathrm{a}$ & 97 a & $100 \mathrm{a}$ & $95 \mathrm{a}$ & 97 a & $100 \mathrm{a}$ & $92 \mathrm{ab}$ & $98 \mathrm{ab}$ & $100 \mathrm{a}$ \\
\hline $\begin{array}{l}\text { Glyphosate } / S \text {-metolachlor/mesotrione }+ \\
\text { atrazine }\end{array}$ & $1050 / 1050 / 105+280$ & $97 \mathrm{a}$ & $97 \mathrm{ab}$ & $100 \mathrm{a}$ & 94 a & $97 \mathrm{ab}$ & $100 \mathrm{a}$ & $92 \mathrm{ab}$ & $98 \mathrm{ab}$ & $100 \mathrm{a}$ \\
\hline Glyphosate + mesotrione + atrazine & $900+100+280$ & $98 \mathrm{a}$ & $98 \mathrm{a}$ & $100 \mathrm{ab}$ & 95 a & 99 a & $100 \mathrm{a}$ & $95 \mathrm{ab}$ & $99 \mathrm{ab}$ & $100 \mathrm{a}$ \\
\hline $\begin{array}{l}\text { Glyphosate }+S \text {-metolachlor/atrazine/ } \\
\text { mesotrione }\end{array}$ & $900+1393 / 524 / 139$ & $99 a$ & 99 a & $100 \mathrm{a}$ & 98 a & 99 a & $100 \mathrm{a}$ & $97 \mathrm{ab}$ & $99 a$ & $100 \mathrm{a}$ \\
\hline $\begin{array}{l}\text { Glyphosate }+S \text {-metolachlor/mesotrione/ } \\
\text { bicyclopyrone/atrazine }\end{array}$ & $900+1259 / 140 / 35 / 588$ & 99 a & 99 a & $100 \mathrm{ab}$ & $99 a$ & 99 a & $100 \mathrm{a}$ & 99 a & $100 \mathrm{a}$ & $100 \mathrm{a}$ \\
\hline
\end{tabular}

Note: Herbicide treatments glyphosate + mesotrione + atrazine and glyphosate $/ S$-metolachlor/mesotrione + atrazine included Agral ${ }^{\circledR} 90($ Syngenta Canada Inc., 140 Research Lane, Research Park, Guelph, ON.) $(0.2 \% \mathrm{v} / \mathrm{v})$; and glyphosate + tolpyralate + atrazine included methylated seed oil (MSO Concentrate $\left.{ }^{\circledR}\right)$ (Loveland Products Inc., 3005 Rocky Mountain Ave., Loveland, CO.) (0.5\% v/v), and urea ammonium nitrate (UAN 28-0-0) (Sylvite, 3221 North Service Road, Burlington, ON.) $(2.5 \% \mathrm{v} / \mathrm{v})$. a-c: Means followed by the same letter are not significantly different $(\mathrm{P}<0.05)$.

Table 4. Density and biomass of multiple-herbicide-resistant (MHR) waterhemp 4 weeks after EPOST application (WAA) in corn treated with herbicide tank-mixtures applied EPOST from five field trials conducted in Ontario, Canada in 2019 and 2020.

\begin{tabular}{|c|c|c|c|c|c|c|c|}
\hline \multirow{2}{*}{ Treatment $^{\dagger}$} & \multirow{2}{*}{$\begin{array}{c}\text { Rate } \\
\left(\mathrm{g} \text { ae or ai ha }{ }^{-1}\right)\end{array}$} & \multicolumn{3}{|c|}{ Density (plants $\mathrm{m}^{-2}$ ) and site } & \multicolumn{3}{|c|}{ Biomass $\left(\mathrm{g} \cdot \mathrm{m}^{-2}\right)$ and site } \\
\hline & & $\mathrm{S} 4$ & S1, S3 & $\mathrm{S} 2, \mathrm{~S} 5$ & S4 & $\mathrm{S} 1, \mathrm{~S} 3$ & S2, S5 \\
\hline Weed-free control & - & 0 & 0 & 0 & 0 & 0 & 0 \\
\hline Nontreated control & - & $962 \mathrm{a}$ & $263 \mathrm{a}$ & $60 \mathrm{a}$ & $259.4 \mathrm{a}$ & $70.2 \mathrm{a}$ & $72.2 \mathrm{a}$ \\
\hline Glyphosate $+S$-metolachlor/atrazine & $900+1600 / 1280$ & $177 \mathrm{abc}$ & $86 \mathrm{ab}$ & $0 \mathrm{~b}$ & $60.0 \mathrm{~b}$ & $15.7 \mathrm{~b}$ & $0 \mathrm{~b}$ \\
\hline Glyphosate + dicamba/atrazine & $900+504 / 996$ & $246 \mathrm{ab}$ & $45 \mathrm{bc}$ & $0 \mathrm{~b}$ & $28.0 \mathrm{~b}$ & $5.3 \mathrm{bc}$ & $0 \mathrm{~b}$ \\
\hline Glyphosate + tolpyralate + atrazine & $900+40+1120$ & 79 abcd & $1 \mathrm{e}$ & $0 \mathrm{~b}$ & $0.9 \mathrm{c}$ & $0.1 \mathrm{~d}$ & $0 \mathrm{~b}$ \\
\hline Glyphosate + isoxaflutole + atrazine & $900+105+1063$ & 38 bcde & $7 \mathrm{cde}$ & $0 \mathrm{~b}$ & $5.7 \mathrm{bc}$ & $0.5 \mathrm{~d}$ & $0 \mathrm{~b}$ \\
\hline
\end{tabular}




\section{Continued}

\begin{tabular}{|c|c|c|c|c|c|c|c|}
\hline $\begin{array}{l}\text { Glyphosate + topramezone/dimethenamid-P + } \\
\text { dicamba/atrazine }\end{array}$ & $900+12.5 / 630+488 / 966$ & $7 \mathrm{cde}$ & $1 \mathrm{e}$ & $0 \mathrm{~b}$ & $0.8 \mathrm{c}$ & $0 \mathrm{~d}$ & $0 \mathrm{~b}$ \\
\hline Glyphosate + pyroxasulfone + dicamba/atrazine & $900+150+488 / 966$ & 27 bcde & $19 \mathrm{bcd}$ & $0 \mathrm{~b}$ & $6.6 \mathrm{bc}$ & $1.4 \mathrm{~cd}$ & $0 \mathrm{~b}$ \\
\hline Glyphosate/2,4-D choline + rimsulfuron + mesotrione & $563 / 591+15+144$ & $9 \mathrm{cde}$ & $1 \mathrm{de}$ & $0 \mathrm{~b}$ & $0.7 \mathrm{c}$ & $0 \mathrm{~d}$ & $0 \mathrm{~b}$ \\
\hline Glyphosate $+S$-metolachlor/mesotrione/bicyclopyrone & $900+1268 / 141 / 35$ & 37 bcde & 10 cde & $0 \mathrm{~b}$ & $2.0 \mathrm{c}$ & $0.6 \mathrm{~d}$ & $0 \mathrm{~b}$ \\
\hline Glyphosate/ $S$-metolachlor/mesotrione + atrazine & $1050 / 1050 / 105+280$ & 10 cde & $5 \mathrm{cde}$ & $0 \mathrm{~b}$ & $2.1 \mathrm{c}$ & $0.4 \mathrm{~d}$ & $0 \mathrm{~b}$ \\
\hline Glyphosate + mesotrione + atrazine & $900+100+280$ & $10 \mathrm{cde}$ & $7 \mathrm{cde}$ & $0 \mathrm{~b}$ & $0.1 \mathrm{c}$ & $0.3 \mathrm{~d}$ & $0 \mathrm{~b}$ \\
\hline Glyphosate $+S$-metolachlor/atrazine/mesotrione & $900+1393 / 524 / 139$ & $2 \mathrm{de}$ & $1 \mathrm{de}$ & $0 \mathrm{~b}$ & $0.1 \mathrm{c}$ & $0.1 \mathrm{~d}$ & $0 \mathrm{~b}$ \\
\hline $\begin{array}{l}\text { Glyphosate/2,4-D choline + rimsulfuron + mesotrione } \\
\text { + atrazine }\end{array}$ & $563 / 591+15+144+1008$ & $3 \mathrm{de}$ & $0 \mathrm{e}$ & $0 \mathrm{~b}$ & $0.5 \mathrm{c}$ & $0 \mathrm{~d}$ & $0 \mathrm{~b}$ \\
\hline $\begin{array}{l}\text { Glyphosate }+S \text {-metolachlor/mesotrione/ } \\
\text { bicyclopyrone/atrazine }\end{array}$ & $900+1259 / 140 / 35 / 588$ & $1 \mathrm{e}$ & $1 \mathrm{de}$ & $0 \mathrm{~b}$ & $0.2 \mathrm{c}$ & $0 \mathrm{~d}$ & $0 \mathrm{~b}$ \\
\hline
\end{tabular}

Note: Herbicide treatments glyphosate + mesotrione + atrazine and glyphosate $/ S$-metolachlor/mesotrione + atrazine included Agral ${ }^{\circledR} 90($ Syngenta Canada Inc., 140 Research Lane, Research Park, Guelph, ON.) $(0.2 \% \mathrm{v} / \mathrm{v})$; and glyphosate + tolpyralate + atrazine included methylated seed oil (MSO Concentrate $\left.{ }^{\circledR}\right)$ (Loveland Products Inc., 3005 Rocky Mountain Ave., Loveland, CO.) (0.5\% v/v), and urea ammonium nitrate (UAN 28-0-0) (Sylvite, 3221 North Service Road, Burlington, ON.) $(2.5 \% \mathrm{v} / \mathrm{v})$. a-d: Means followed by the same letter are not significantly different $(\mathrm{P}<0.05)$.

Table 5. Effect of herbicide tank-mixtures applied EPOST on corn injury [1, 2 and 4 weeks after the EPOST application (WAA)] and yield from five field trials conducted in Ontario, Canada in 2019 and 2020.

\begin{tabular}{|c|c|c|c|c|c|c|c|}
\hline \multirow{3}{*}{ Treatment $^{\dagger}$} & \multirow{3}{*}{ Rate (g ae or ai ha ${ }^{-1}$ ) } & \multicolumn{3}{|c|}{ Injury (\%) } & & & \\
\hline & & \multirow{2}{*}{$\frac{1 \mathrm{WAA}}{\mathrm{S} 2}$} & \multirow{2}{*}{$\frac{2 \mathrm{WAA}}{\mathrm{S} 2}$} & \multirow{2}{*}{$\frac{4 \mathrm{WAA}}{\mathrm{S} 2}$} & \multicolumn{3}{|c|}{ Yield $\left(t \cdot h a^{-1}\right)$ and site } \\
\hline & & & & & S4 & S1, S3 & S2, S5 \\
\hline Weed-free control & - & - & - & - & $8.0 \mathrm{a}$ & $8.4 \mathrm{a}$ & $8.7 \mathrm{ab}$ \\
\hline Nontreated control & - & - & - & - & $4.9 \mathrm{~b}$ & $7.3 \mathrm{a}$ & $8.3 \mathrm{ab}$ \\
\hline Glyphosate $+S$-metolachlor/atrazine & $900+1600 / 1280$ & $1 \mathrm{a}$ & $0 \mathrm{a}$ & $0 \mathrm{a}$ & $7.9 \mathrm{a}$ & $8.0 \mathrm{a}$ & $9.4 \mathrm{a}$ \\
\hline Glyphosate + dicamba/atrazine & $900+504 / 996$ & $0 \mathrm{a}$ & $0 \mathrm{a}$ & $0 \mathrm{a}$ & $8.5 \mathrm{a}$ & $8.6 \mathrm{a}$ & $8.7 \mathrm{ab}$ \\
\hline Glyphosate + tolpyralate + atrazine & $900+40+1120$ & $8 \mathrm{ab}$ & $4 \mathrm{a}$ & $1 \mathrm{a}$ & $8.8 \mathrm{a}$ & $8.2 \mathrm{a}$ & $8.1 \mathrm{ab}$ \\
\hline Glyphosate + isoxaflutole + atrazine & $900+105+1063$ & $0 \mathrm{a}$ & $0 \mathrm{a}$ & $0 \mathrm{a}$ & $8.4 \mathrm{a}$ & $8.0 \mathrm{a}$ & $9.2 \mathrm{ab}$ \\
\hline $\begin{array}{l}\text { Glyphosate + topramezone/dimethenamid-P + } \\
\text { dicamba/atrazine }\end{array}$ & $900+12.5 / 630+488 / 966$ & $1 \mathrm{a}$ & $1 \mathrm{a}$ & $1 \mathrm{a}$ & $8.2 \mathrm{a}$ & $7.6 \mathrm{a}$ & $8.5 \mathrm{ab}$ \\
\hline Glyphosate + pyroxasulfone + dicamba/atrazine & $900+150+488 / 966$ & $3 \mathrm{a}$ & $3 \mathrm{a}$ & $3 \mathrm{a}$ & $7.9 \mathrm{a}$ & $8.0 \mathrm{a}$ & $8.5 \mathrm{ab}$ \\
\hline Glyphosate/2,4-D choline + rimsulfuron + mesotrione & $563 / 591+15+144$ & $3 \mathrm{a}$ & $6 \mathrm{a}$ & $5 \mathrm{a}$ & $8.6 \mathrm{a}$ & $8.5 \mathrm{a}$ & $8.3 \mathrm{ab}$ \\
\hline Glyphosate $+S$-metolachlor/mesotrione/bicyclopyrone & $900+1268 / 141 / 35$ & $0 \mathrm{a}$ & $0 \mathrm{a}$ & $0 \mathrm{a}$ & $8.2 \mathrm{a}$ & $8.0 \mathrm{a}$ & $8.7 \mathrm{ab}$ \\
\hline Glyphosate/ $S$-metolachlor/mesotrione + atrazine & $1050 / 1050 / 105+280$ & $0 \mathrm{a}$ & $0 \mathrm{a}$ & $0 \mathrm{a}$ & $9.2 \mathrm{a}$ & $8.1 \mathrm{a}$ & $8.5 \mathrm{ab}$ \\
\hline Glyphosate + mesotrione + atrazine & $900+100+280$ & $0 \mathrm{a}$ & $0 \mathrm{a}$ & $0 \mathrm{a}$ & $9.8 \mathrm{a}$ & $8.9 \mathrm{a}$ & $9.0 \mathrm{ab}$ \\
\hline Glyphosate $+S$-metolachlor/atrazine/mesotrione & $900+1393 / 524 / 139$ & $0 \mathrm{a}$ & $0 \mathrm{a}$ & $0 \mathrm{a}$ & $8.3 \mathrm{a}$ & $8.6 \mathrm{a}$ & $8.5 \mathrm{ab}$ \\
\hline $\begin{array}{l}\text { Glyphosate/2,4-D choline + rimsulfuron + mesotrione } \\
\text { + atrazine }\end{array}$ & $563 / 591+15+144+1008$ & $28 \mathrm{~b}$ & $31 \mathrm{~b}$ & $31 \mathrm{~b}$ & $8.2 \mathrm{a}$ & $8.5 \mathrm{a}$ & $7.3 \mathrm{~b}$ \\
\hline $\begin{array}{l}\text { Glyphosate }+S \text {-metolachlor/mesotrione/ } \\
\text { bicyclopyrone/atrazine }\end{array}$ & $900+1259 / 140 / 35 / 588$ & $0 \mathrm{a}$ & $0 \mathrm{a}$ & $0 \mathrm{a}$ & $8.8 \mathrm{a}$ & $8.5 \mathrm{a}$ & $8.4 \mathrm{ab}$ \\
\hline
\end{tabular}

Note: Herbicide treatments glyphosate + mesotrione + atrazine and glyphosate/ $S$-metolachlor/mesotrione + atrazine included Agral ${ }^{\circledR} 90($ Syngenta Canada Inc., 140 Research Lane, Research Park, Guelph, ON.) $(0.2 \% \mathrm{v} / \mathrm{v})$; and glyphosate + tolpyralate + atrazine included methylated seed oil (MSO Concentrate $\left.{ }^{\circledR}\right)$ (Loveland Products Inc., 3005 Rocky Mountain Ave., Loveland, CO) (0.5\% v/v), and urea ammonium nitrate (UAN 28-0-0) (Sylvite, 3221 North Service Road, Burlington, ON.) (2.5\% v/v). a-b: Means followed by the same letter are not significantly different $(\mathrm{P}<0.05)$. 
At S1, S3, and S4, glyphosate $+S$-metolachlor/mesotrione/bicyclopyrone/ atrazine, glyphosate/2,4-D choline + rimsulfuron + mesotrione + atrazine, glyphosate $+S$-metolachlor/atrazine/mesotrione, and glyphosate + mesotrione + atrazine controlled MHR waterhemp $95 \%$ to $100 \%$ at 4,8 , and 12 WAA and reduced plant density and biomass by $97 \%$ to $100 \%$. Glyphosate/ $S$-metolachlor/ mesotrione + atrazine, glyphosate $+S$-metolachlor/mesotrione/bicyclopyrone, glyphosate/2,4-D choline + rimsulfuron + mesotrione, and glyphosate + pyroxasulfone + dicamba/atrazine controlled MHR waterhemp $90 \%$ to $99 \%$ at 4,8 , and 12 WAA and reduced plant density and biomass $96 \%$ to $100 \%$. Control of MHR waterhemp ranged from $99 \%$ to $100 \%$ and plant density and biomass were reduced $100 \%$ with all herbicides at S2 and S5. These findings are consistent with Sarangi and Jhala [46] who reported excellent control of MHR waterhemp with $S$-metolachlor/mesotrione/bicyclopyrone/atrazine applied POST to 8 to $10 \mathrm{~cm}$ and 15 to $18 \mathrm{~cm}$ waterhemp. Benoit et al. [3] [19] [33] and Vyn et al. [31] reported comparable control of MHR waterhemp with mesotrione + atrazine applied POST providing $\geq 90 \%$ control 4,8 and 12 WAA in multiple trials. Vyn et al. [31] reported similar control of MHR waterhemp with 2,4-D/atrazine (1404 g.ha ${ }^{-1}$ ) of $84 \%$ to $100 \% 4$ and 10 WAA. Additionally, Sarangi et al. [47] and Chahal et al. [45] reported excellent control of MHR waterhemp with glyphosate $+2,4$-D choline $\left(840+800 \mathrm{~g} \cdot \mathrm{ha}^{-1}\right)$ applied POST, however, Chahal et al. [45] suggested that plants should be less than $10 \mathrm{~cm}$ in height at application. At S1, S3, and S4, glyphosate + topramezone/dimethenamid-P + dicamba/atrazine, glyphosate + isoxaflutole + atrazine, and glyphosate + tolpyralate + atrazine controlled MHR waterhemp $79 \%$ to $99 \%$ at 4,8 and 12 WAA and reduced plant density and biomass $92 \%$ to $100 \%$. These results complement another Ontario study that reported $\geq 96 \%$ MHR waterhemp control from 4 to 12 WAA with glyphosate + tolpyralate + atrazine $\left(900+30+560 \mathrm{~g} \cdot \mathrm{ha}^{-1}\right)$ applied POST [26]. Moreover, MHR waterhemp control with isoxaflutole + atrazine applied EPOST was similar to PRE applications that provided $82 \%$ to $100 \%$ control in other studies [16] [19] [31] [48]. Control of MHR waterhemp was similar for all herbicide tank-mixtures except glyphosate $+S$-metolachlor/atrazine and glyphosate + dicamba/atrazine at S4, S1 and S3 which resulted in the lowest level of control ranging from $73 \%$ to $75 \%$ and $76 \%$ to $83 \%$, respectively. Glyphosate + $S$-metolachlor/atrazine resulted in lower MHR waterhemp control than glyphosate + dicamba/atrazine at S1 and S3; however, control was similar at S4. This is consistent with Benoit et al. [19] who reported 84\% and 87\% MHR waterhemp control 4 and 8 WAA with dicamba/atrazine POST. In contrast, Soltani et al. [16] reported greater MHR waterhemp control and reductions in density and biomass with dicamba/atrazine $\left(1800 \mathrm{~g} \cdot \mathrm{ha}^{-1}\right)$ POST that were similar to mesotrione + atrazine POST and isoxaflutole + atrazine PRE in that study. Schryver et al. [34] reported MHR waterhemp control with dicamba $\left(600 \mathrm{~g} \cdot \mathrm{ha}^{-1}\right)$ is greatest when applied POST resulting in $91 \%$ to $100 \%$ control compared to $60 \%$ to $65 \%$ at 10 WAA when applied PRE. Lower control with glyphosate + dicam- 
ba/atrazine may be due to lower dicamba application rate $\left(504 \mathrm{~g} \cdot \mathrm{ha}^{-1}\right)$. Control of MHR waterhemp from 4 to $12 \mathrm{WAA}$ increased at S1 and S3 and decreased at S4 for most herbicide tank-mixtures evaluated. Increasing MHR waterhemp control over the course of the growing season could be due to natural thinning of waterhemp populations reported by Benoit et al. [19] and [49]; in contrast, late emerging cohorts have been reported to reduce end-of-season control as well [2].

Corn injury was $\leq 10 \%$ for all herbicide treatments 1,2 , and 4 WAA at all sites except S2 (Table 5). Glyphosate/2,4-D choline + rimsulfuron + mesotrione + atrazine caused $28 \%, 31 \%$, and $31 \%$ corn injury 1,2 , and 4 WAA, respectively at S2; symptoms included brace root malformation and lodging which resulted in reduced corn stand. Applications of glyphosate/2,4-D choline to non-ENLIST ${ }^{\mathrm{TM}}$ hybrids can cause stalk brittleness, leaning, malformed brace roots, and leaf rolling in the whorl [50] [51]. Ruen et al. [38] reported similar leaf necrosis and leaning of ENLIST ${ }^{\mathrm{TM}}$ corn hybrids treated with single applications of glyphosate/2,4-D choline at V4 and V7 corn growth stages and sequential applications at V4 $f b$ V7. Interestingly, the addition of atrazine to glyphosate/2,4-D choline + rimsulfuron + mesotrione increased corn injury $25 \%, 25 \%$, and $26 \%$ at 1,2 , and $4 \mathrm{WAA}$, respectively. We do not have an explanation for this observation; the response should be evaluated in future studies to determine if this is a real response. Tolerance of conventional corn hybrids to 2,4-D varies with hybrid, corn growth stage at application, soil characteristics, and weather conditions [38]. It is recommended that glyphosate/2,4-D choline (ENLIST DUO) only be applied to ENLIST $^{\mathrm{TM}}$ field corn hybrids that contain the AAD-1 transgene [35]. Glyphosate/2,4-D choline applications can also be made up to the V8 corn growth stage; in this study, herbicides were applied to V4 corn (data not shown). Corn injury caused by glyphosate/2,4-D choline + rimsulfuron + mesotrione + atrazine resulted in lower corn yield than glyphosate $+S$-metolachlor/atrazine; however, yield was similar to the weed-free control. When waterhemp was left uncontrolled, corn yield was reduced $39 \%$ at $S 4$ and was similar to another Ontario study that reported a corn yield reduction of $48 \%$ [16]. Relative to the weed-free control, corn yield was not reduced at S1, S2, S3, and S5 which could again be the result of comparatively lower MHR waterhemp density and biomass. Cordes et al. [14] reported corn yield reductions due to the late removal of waterhemp when plants reached $15 \mathrm{~cm}$. This result supports previous research that suggests EPOST herbicide applications reduce early season weed interference and minimize corn yield loss [13].

\section{Conclusion}

Most of the EPOST herbicide tank-mixtures evaluated in this study resulted in greater than $90 \%$ control of MHR waterhemp. The top four herbicides treatments were glyphosate $+S$-metolachlor/mesotrione/bicyclopyrone/atrazine, glyphosate/2,4-D choline + rimsulfuron + mesotrione + atrazine, glyphosate + 
$S$-metolachlor/atrazine/mesotrione, and glyphosate + mesotrione + atrazine which controlled MHR waterhemp $95 \%$ to $100 \%$ throughout the growing season at all sites. Glyphosate/S-metolachlor/mesotrione + atrazine, glyphosate + $S$-metolachlor/mesotrione/bicyclopyrone, glyphosate/2,4-D choline + rimsulfuron + mesotrione, and glyphosate + pyroxasulfone + dicamba/atrazine provided $90 \%$ to $100 \%$ MHR waterhemp control 4, 8, and 12 WAA. Control of MHR waterhemp was similar amongst all herbicide tank-mixtures, except glyphosate + $S$-metolachlor/atrazine and glyphosate + dicamba/atrazine which resulted in lower control than all other treatments, control ranged from $61 \%$ to $100 \%$ and $63 \%$ to $100 \%$, respectively. Reductions in MHR waterhemp control with these herbicides resulted in greater MHR waterhemp density and biomass 4 WAA at three of five sites. Differences in MHR waterhemp control were the result of variation in population resistance profiles, competitiveness of individual populations, and comparatively lower MHR waterhemp density and biomass at two of five sites. Furthermore, herbicide tank-mixtures reduced MHR waterhemp density and biomass $>91 \%$ and $97 \%$, respectively, except glyphosate $+S$-metolachlor/ atrazine and glyphosate + dicamba/atrazine (67\% to $82 \%$ and $74 \%$ to $92 \%)$. Weed interference caused by MHR waterhemp did not reduce corn yield in this study at four out of five sites. This study identifies EPOST herbicide tank-mixtures that expand POST MHR waterhemp management and have a wide margin of crop safety. Corn producers should incorporate the use of strategic tillage, cover crops, crop row spacing, crop density, PRE herbicides, EPOST herbicides, POST herbicides, and PRE $f b$ POST herbicide programs to steward the use of currently effective MOA and reduce the evolution of herbicide resistance. Strategies to manage MHR waterhemp should be implemented early in the growing season, herbicide applications should be made before $M H R$ waterhemp reaches $10 \mathrm{~cm}$ in height to prevent corn yield loss.

\section{Acknowledgements}

The authors acknowledge Chris Kramer for his technical support, Dr. Michelle Edwards for her statistical support, the University of Guelph, Ridgetown Campus summer staff for their field support and the Grain Farmers of Ontario and herbicide manufacturers for providing funding for this research.

\section{Conflicts of Interest}

The authors declare no conflicts of interest regarding the publication of this paper.

\section{References}

[1] Green, J.M. and Owen, M.D. (2011) Herbicide-Resistant Crops: Utilities and Limitations for Herbicide-Resistant Weed Management. Journal of Agricultural and Food Chemistry, 59, 5819-5829. https://doi.org/10.1021/jf101286h

[2] Steckel, L.E. and Sprague, C.L. (2004) Common Waterhemp (Amaranthus rudis) 
Interference in Corn. Weed Science, 52, 359-364.

https://doi.org/10.1614/WS-03-066R1

[3] Benoit, L., Soltani, N., Hooker, D.C., Robinson, D.E. and Sikkema, P.H. (2019b) Efficacy of HPPD-Inhibiting Herbicides Applied Preemergence or Postemergence for Control of Multiple Herbicide Resistant Waterhemp [Amaranthus tuberculatus (Moq.) Sauer]. Canadian Journal of Plant Science, 99, 379-383.

https://doi.org/10.1139/cjps-2018-0320

[4] Heap, I. (2020) The International Survey of Herbicide Resistant Weeds. http://www.weedscience.org

[5] Costea, M., Weaver, S.E. and Tardif, F.J. (2005) The Biology of Invasive Alien Plants in Canada. 3. Amaranthus tuberculatus (Moq.) Sauer var. rudis (Sauer) Costea \& Tardif. Canadian Journal of Plant Science, 85, 507-522. https://doi.org/10.4141/P04-101

[6] Hartzler, R.G., Buhler, D.D. and Stoltenberg, D.E. (1999) Emergence Characteristics of Four Annual Weed Species. Weed Science, 47, 578-584. https://doi.org/10.1017/S0043174500092298

[7] Horak, M.J. and Loughin, T.M. (2000) Growth Analysis of Four Amaranthus Species. Weed Science, 48, 347-355. https://doi.org/10.1614/0043-1745(2000)048[0347:GAOFAS]2.0.CO;2

[8] Schleufer, I.L., Roeth, F.W. and Mortensen, D.A. (1992) Triazine Resistant Amaranthus Control. Proceeding of the North Central Weed Science Society, 47, 20-21.

[9] Shoup, D.E., Al-Khatib, K. and Peterson, D.E. (2003) Common Waterhemp (Amaranthus rudis) Resistance to Protoporphyrinogen Oxidase-Inhibiting Herbicides. Weed Science, 51, 145-150. https://doi.org/10.1614/0043-1745(2003)051[0145:CWARRT]2.0.CO;2

[10] Shergill, L., Barlow, B., Bish, M. and Bradley, K. (2018) Investigations of 2,4-D and Multiple Herbicide Resistance in a Missouri Waterhemp (Amaranthus tuberculatus) Population. Weed Science, 66, 386-394. https://doi.org/10.1017/wsc.2017.82

[11] Strom, S.A., Gonzini, L.C., Mitsdarfer, C., Davis, A.S., Riechers, D.E. and Hager, A.G. (2019) Characterization of Multiple Herbicide-Resistant Waterhemp (Amaranthus tuberculatus) Populations from Illinois to VLCFA-Inhibiting Herbicides. Weed Science, 67, 369-379. https://doi.org/10.1017/wsc.2019.13

[12] Bell, M.S., Hager, A.G. and Tranel, P.J. (2013) Multiple Resistance to Herbicides from Four Environment-of-Action Groups in Waterhemp (Amaranthus tuberculatus). Weed Science, 61, 460-468. https://doi.org/10.1614/WS-D-12-00166.1

[13] Hall, M.R., Swanton, C.J. and Anderson, G.W. (1992) The Critical Period of Weed Control in Grain Corn (Zea mays). Weed Science, 40, 441-447. https://doi.org/10.1017/S0043174500051882

[14] Cordes, J.C., Johnson, W.G., Scharf, P. and Smeda, R.J. (2004) Late-Emerging Common Waterhemp (Amaranthus rudis) Interference in Conventional Tillage Corn. Weed Technology, 18, 999-1005. https://doi.org/10.1614/WT-03-185R

[15] Nordby, D.E. and Hartzler, R.G. (2004) Influence of Corn on Common Waterhemp (Amaranthus rudis) Growth and Fecundity. Weed Science, 52, 255-259. https://doi.org/10.1614/WS-03-060R

[16] Soltani, N., Vyn, J.D. and Sikkema, P.H. (2009) Control of Common Waterhemp (Amaranthus tuberculatus var. rudis) in Corn and Soybean with Sequential Herbicide Applications. Canadian Journal of Plant Science, 89, 127-132.

https://doi.org/10.4141/CJPS08051 
[17] Cox, W.J., Hahn, R.R. and Stachowski, P.J. (2006) Time of Weed Removal with Glyphosate Affects Corn Growth and Yield Components. Agronomy Journal, 98, 349-353. https://doi.org/10.2134/agronj2005.0078

[18] Knezevic, S.Z., Evans, S.P., Blankenship, E.E., Van Acker, R.C. and Lindquist, J.L. (2002) Critical Period of Weed Control: The Concept and Data Analysis. Weed Science, 50, 773-786. https://doi.org/10.1614/0043-1745(2002)050[0773:CPFWCT]2.0.CO;2

[19] Benoit, L., Soltani, N., Hooker, D.C., Robinson, D.E. and Sikkema, P.H. (2019) Control of Multiple-Resistant Waterhemp [Amaranthus tuberculatus (Moq.) Sauer] with Preemergence and Postemergence Herbicides in Corn in Ontario. Canadian Journal of Plant Science, 99, 364-370. https://doi.org/10.1139/cjps-2018-0087

[20] Hedges, B.K., Soltani, N., Hooker, D.C., Robinson, D.E. and Sikkema, P.H. (2018) Control of Glyphosate-Resistant Waterhemp with Two-Pass Weed Control Strategies in Glyphosate/Dicamba-Resistant Soybean. American Journal of Plant Sciences, 9, 1424-1432. https://doi.org/10.4236/ajps.2018.97104

[21] Jhala, A.J., Sandell, L.D., Sarangi, D., Kruger, G.R. and Knezevic, S.Z. (2017) Control of Glyphosate-Resistant Common Waterhemp (Amaranthus rudis) in Glufosinate-Tolerant Soybean. Weed Technology, 31, 32-45.

https://doi.org/10.1017/wet.2016.8

[22] Mitchell, G., Bartlett, D.W., Fraser, T.E.M., Hawkes, T.R., Holt, D.C., Townson, J.K. and Wichert, R.A. (2001) Mesotrione: A New Selective Herbicide for Use in Maize. Pest Management Science, 57, 120-128. https://doi.org/10.1002/1526-4998(200102)57:2<120::AID-PS254>3.0.CO;2-E

[23] Osipitan, O.A., Scott, J.E. and Knezevic, S.Z. (2018) Tolpyralate Applied Alone and with Atrazine for Weed Control in Corn. The Journal of Agricultural Science, 10, 32-39. https://doi.org/10.5539/jas.v10n10p32

[24] Williams, M.M., Boydston, R.A., Peachey, R.E. and Robinson, D. (2011) Significance of Atrazine as a Tank-Mix Partner with Tembotrione. Weed Technology, 25, 299-302. https://doi.org/10.1614/WT-D-10-00140.1

[25] Hugie, J.A., Bollero, G.A., Tranel, P.J. and Riechers, D.E. (2008) Defining the Rate Requirements for Synergism between Mesotrione and Atrazine in Redroot Pigweed (Amaranthus retroflexus). Weed Science, 56, 265-270.

https://doi.org/10.1614/WS-07-128.1

[26] Khort, J.R. and Sprague, C.L. (2017) Response of a Multiple-Resistant Palmer Amaranth (Amaranthus palmeri) Population to Four HPPD-Inhibiting Herbicides Applied Alone and with Atrazine. Weed Science, 65, 534-535. https://doi.org/10.1017/wsc.2017.28

[27] Woodyard, A.J., Bollero, G.A. and Riechers, D.E. (2009) Broadleaf Weed Management in Corn Utilizing Synergistic Postemergence Herbicide Combinations. Weed Technology, 23, 513-518. https://doi.org/10.1614/WT-08-188.1

[28] Armel, G.R., Richardson, R.J., Wilson, H.P. and Hines, T.E. (2009) Strategies for Control of Horseweed (Conyza canadensis) and Other Winter Annual Weeds in No-Till Corn. Weed Technology, 23, 379-383. https://doi.org/10.1614/WT-08-094.1

[29] Hankamer, B., Barber, J. and Boekema, E.J. (1997) Structure and Membrane Organization of Photosystem II in Green Plants. Annual Review of Plant Physiology and Plant Molecular Biology, 48, 641-671.

https://doi.org/10.1146/annurev.arplant.48.1.641

[30] Anderson, D.D., Higley, L.G., Martin, A.R. and Roeth, F.W. (1996) Competition 
between Triazine-Resistant and Susceptible Common Waterhemp (Amaranthus rudis). Weed Science, 44, 853-859. https://doi.org/10.1017/S0043174500094820

[31] Vyn, J.D., Swanton, C.J., Weaver, S.E. and Sikkema, P.H. (2006) Control of Amaranthus tuberculatus var. rudis (Common Waterhemp) with Pre and Post-Emergence Herbicides in Zea mays L. (Maize). Crop Protection, 25, 1051-1056.

https://doi.org/10.1016/j.cropro.2006.01.016

[32] Munro, I.C., Carlo, G.L., ORR, J.C., Sund, K.G., Wilson, R.M., Kennepohl, E., Lynch, B.S., Jablinske, M. and Lee, N.L. (1992) A Comprehensive, Integrated Review and Evaluation of the Scientific Evidence Relating to the Safety of the Herbicide 2,4-D. Journal of the American College of Toxicology, 11, 559-604. https://doi.org/10.3109/10915819209141893

[33] Benoit, L., Hedges, B., Schryver, M.G., Soltani, N., Hooker, D.C., Robinson, D.E., Laforest, M., Soufiane, B., Tranel, P.J., Giacomini, D. and Sikkema, P.H. (2020) The First Record of Protoporphyrinogen Oxidase and Four-Way Herbicide Resistance in Eastern Canada. Canadian Journal of Plant Science, 100, 327-331. https://doi.org/10.1139/cjps-2018-0326

[34] Schryver, M.G., Soltani, N., Hooker, D.C., Robinson, D.E., Tranel, P.J. and Sikkema, P.H. (2017) Control of Glyphosate-Resistant Waterhemp (Amaranthus tuberculatus var rudis) with Dicamba and Dimethenamid-P in Ontario. Canadian Journal of Plant Science, 98, 362-369. https://doi.org/10.1139/CJPS-2017-0052

[35] Anonymous (2018) ENLIST DUO ${ }^{\mathrm{TM}}$ Herbicide Label. Corteva Agriscience, Wilmington.

[36] Robinson, A.P., Simpson, D.M. and Johnson, W.G. (2012) Summer Annual Weed Control with 2,4-D and Glyphosate. Weed Technology, 26, 657-660. https://doi.org/10.1614/WT-D-12-00081.1

[37] Ontario Ministry of Agriculture, Food and Rural Affairs (OMAFRA) (2018) Publication 75A: Guide to Weed Control in Field Crops 2018. Queen's Printer, Toronto.

[38] Ruen, D.C., Scherder, E.F., Ditmarsen, S.C., Prasifka, P.L., Ellis, J.M., Simpson, D.M., Gallup, C.A. and Hopkins, B.W. (2017) Tolerance of Corn with Glyphosate Resistance and the Aryloxyalkanoate Dioxygenase Trait (AAD-1) to 2,4-D Choline and Glyphosate. Weed Technology, 31, 217-224.

https://doi.org/10.1017/wet.2016.20

[39] Miller, R.M. and Norsworthy, J.K. (2016) Evaluation of Herbicide Programs for Use in a 2,4-D-Resistant Soybean Technology for Control of Glyphosate-Resistant Palmer Amaranth (Amaranthus palmeri). Weed Technology, 30, 366-376. https://doi.org/10.1614/WT-D-15-00129.1

[40] Oliveira, M.C., Jhala, A.J., Gains, T., Irmak, S., Amundsen, K., Scott, J.E. and Knezevic, S.Z. (2017) Confirmation and Control of HPPD-Inhibiting Herbicide-Resistant Waterhemp (Amaranthus tuberculatus) in Nebraska. Weed Technology, 31, 67-79. https://doi.org/10.1017/wet.2016.4

[41] Sarangi, D., Sandell, L.D., Knezevic, S.Z., Aulakh, J.S., Lindquist, J.L., Irmak, S. and Jhala, A.J. (2015) Confirmation and Control of Glyphosate-Resistant Common Waterhemp (Amaranthus rudis) in Nebraska. Weed Technology, 29, 82-92. https://doi.org/10.1614/WT-D-14-00090.1

[42] Hager, A.G., Wax, L.M., Bollero, G.A. and Stoller, E.W. (2003) Influence of Diphenyl Ether Herbicide Application Rate and Timing on Common Waterhemp (Amaranthus rudis) Control in Soybean (Glycine max). Weed Technology, 17, 14-20. https://doi.org/10.1614/0890-037X(2003)017[0014:IODHAR]2.0.CO;2 
[43] Hedges, B.K., Soltani, N., Robinson, D.E., Hooker, D.C. and Sikkema, P.H. (2019) Influence of Glyphosate/Dicamba Application Rate and Timing on the Control of Glyphosate-Resistant Waterhemp in Glyphosate/Dicamba-Resistant Soybean. Canadian Journal of Plant Science, 99, 371-374.

https://doi.org/10.1139/cjps-2018-0101

[44] Legleiter, T.R. and Bradley, K.W. (2008) Glyphosate and Multiple Herbicide Resistance in Common Waterhemp (Amaranthus rudis) Populations from Missouri. Weed Science, 56, 582-587. https://doi.org/10.1614/WS-07-204.1

[45] Chahal, P.S., Aulakh, J.S., Rosenbaum, K. and Jhala, A.J. (2015) Growth Stage Affects Does Response of Selected Glyphosate-Resistant Weeds to Premix of 2,4-D Choline and Glyphosate (Enlist Duo ${ }^{\mathrm{TM}}$ Herbicide). The Journal of Agricultural Science, 7, 1-10. https://doi.org/10.5539/jas.v7n11p1

[46] Sarangi, D. and Jhala, A.J. (2017) Biologically Effective Rates of a New Premix (Atrazine, Bicyclopyrone, Mesotrione, and $S$-Metolachlor) for Preemergence or Postemergence Control of Common Waterhemp [Amaranthus tuberculatus (Moq.) Sauer var. rudis] in Corn. Canadian Journal of Plant Science, 97, 1075-1089. https://doi.org/10.1139/CJPS-2017-0037

[47] Sarangi, D., Stephens, T., Barker, A.L., Patterson, E.L., Gaines, T.A. and Jhala, A.J. (2019) Protoporphyrinogen Oxidase (PPO) Inhibitor-Resistant Waterhemp (Amaranthus tuberculatus) from Nebraska Is Multiple Herbicide Resistant: Confirmation, Mechanism of Resistance, and Management. Weed Science, 67, 510-520. https://doi.org/10.1017/wsc.2019.29

[48] Langdon, N.M., Soltani, N., Raeder, A.J., Hooker, D.C., Robinson, D.E. and Sikkema, P.H. (2020) Influence of Adjuvants on the Control of Glyphosate-Resistant Canada Fleabane and Waterhemp in Corn with Tolpyralate. American Journal of Plant Sciences, 11, 354-371. https://doi.org/10.4236/ajps.2020.113026

[49] Heneghan, J. and Johnson, W. (2017) The Growth and Development of Five Waterhemp (Amaranthus tuberculatus) Populations in a Common Garden. Weed Science, 65, 247. https://doi.org/10.1017/wsc.2016.20

[50] Rodgers, E.G. (1952) Brittleness and Other Responses of Corn to 2,4-Dichlorophenoxyacetic Acid. Plant Physiology, 27, 153-172. https://doi.org/10.1104/pp.27.1.153

[51] Striegel, A., Lawrence, N.C., Knezevic, S.Z., Krumm, J.T., Hein, G. and Jhala, A.J. (2020) Control of Glyphosate/Glufosinate-Resistant Volunteer Corn in Corn Resistant to Aryloxyphenoxyproprionates. Weed Technology, 34, 309-317.

https://doi.org/10.1017/wet.2020.41 